\title{
Hystereses in dwarf nova outbursts and low-mass X-ray binaries
}

\author{
J.-M. Hameury ${ }^{1}$, J.-P. Lasota ${ }^{2,3}$, C. Knigge ${ }^{4}$, and E. G. Körding ${ }^{5}$ \\ 1 Université de Strasbourg, CNRS, Observatoire Astronomique de Strasbourg, UMR 7550, 67000 Strasbourg, France \\ e-mail: jean-marie.hameury@astro.unistra.fr \\ 2 Institut d'Astrophysique de Paris, CNRS et Sorbonne Universités, UPMC Paris 06, UMR 7095, 98bis bd Arago, 75014 Paris, \\ France \\ ${ }^{3}$ Nicolaus Copernicus Astronomical Center, Polish Academy of Sciences, Bartycka 18, 00-716 Warsaw, Poland \\ 4 School of Physics and Astronomy, University of Southampton, Southampton SO17 1BJ, UK \\ 5 Department of Astrophysics/IMAPP, Radboud University, PO Box 9010, 6500 GL Nijmegen, The Netherlands
}

Received 25 October 2016 / Accepted 2 January 2017

\begin{abstract}
Context. The disc instability model (DIM) successfully explains why many accreting compact binary systems exhibit outbursts during which their luminosity increases by orders of magnitude. The DIM correctly predicts which systems should be transient and works regardless of whether the accretor is a black hole, a neutron star, or a white dwarf. However, it has been known for some time that the outbursts of X-ray binaries, which contain neutron-star or black-hole accretors, exhibit hysteresis in the X-ray hardness-intensity diagram (HID). More recently, it has been shown that the outbursts of accreting white dwarfs also show hysteresis, but in a diagram combining optical, EUV, and X-ray fluxes.

Aims. We examine the nature of the hysteresis observed in cataclysmic variables and low-mass X-ray binaries.

Methods. We used our disc evolution code for modelling dwarf nova outbursts, and constructed the hardness intensity diagram as predicted by the disc instability model.

Results. We show explicitly that the standard DIM, modified only to account for disc truncation, can explain the hysteresis observed in accreting white dwarfs, but cannot explain that observed in X-ray binaries.

Conclusions. The spectral evidence for the existence of different accretion regimes or components (disc, corona, jets, etc.) should only be based on wavebands that are specific to the innermost parts of the discs, i.e. EUV and X-rays; this task is difficult because of interstellar absorption. The existing data, however, indicate that a hysteresis is in the EUV - X-ray domain is present in SS Cyg.
\end{abstract}

Key words. accretion, accretion disks - stars: dwarf novae - X-rays: binaries - instabilities

\section{Introduction}

Dwarf novae (DNs) are a subclass of cataclysmic variables (CVs) that undergo recurrent outbursts usually lasting a few days. Outbursts are separated by quiescent intervals of a few weeks (see e.g. Warner 1995, for an encyclopaedic review of $\mathrm{CVs}$ ). It is now widely accepted that these outbursts are the result of a thermal-viscous instability of the accretion disc that arises when hydrogen becomes partially ionized and the opacities depend sensitively on temperature (see Lasota 2001, for a review of the model). A stability analysis shows that when the local mass transfer rate $\dot{M}$ is in the range $\left[\dot{M}_{\text {crit }}^{-}, \dot{M}_{\text {crit }}^{+}\right]$the disc is thermally and viscously unstable; the disc is stable on a cold branch for lower values of $\dot{M}$ and, the disc is also stable for higher values of $\dot{M}$, but on a hot branch.

In quiescence, mass accumulates in the disc, which slowly heats up, and the local mass transfer rate increases until, at some point in the disc, the rate reaches the critical $\dot{M}_{\text {crit }}^{-}$ for which the instability develops. The instability propagates throughout the disc via two heating fronts moving inwards and outwards; the outer heating front eventually reaches the outer edge of the disc, which is then almost in a steady state with a mass accretion rate onto the white dwarf that is higher than the mass transfer rate from the secondary. The disc empties until a cooling front starts propagating at the outer edge of the disc and brings the system back to quiescence.
Confronting the predictions of the disc instability model (DIM) with the observations has been a long standing endeavour. The objective of this endeavour is to validate the model and also to put constraints on the viscosity, which still remains poorly understood despite spectacular progress, with the realization that angular momentum transport is most probably the result of the magnetorotational instability (MRI; Balbus \& Hawley 1991). In the DIM, viscosity is still modelled according to the Shakura \& Sunyaev (1973) $\alpha$ parametrization. Whereas $\alpha$ is reasonably well constrained when the disc is hot, there are still large uncertainties when the disc is in quiescence. A new DIM version using viscosity and disc vertical structures obtained through MRI simulation has been recently developed by Coleman et al. (2016) but in this case the cold disc physics also causes problems.

Colour variations should be one of the most obvious observational features that models should aim to reproduce. It has long been known that dwarf novae follow a loop in the $U-B$, $B-V$ colour plane (e.g. Bailey 1980, for the cases of SS Cyg and VW Hyi). This fact seems to have been forgotten. This is perhaps because the precise modelling of the optical colours of the accretion disc is not trivial, as this modelling, in principle, requires calculations of detailed spectra. This loop could also be accounted for by the DIM and mass transfer instability model (Bath \& Pringle 1981). A similar phenomenon is also seen when comparing the visible and extreme UV (EUV) or far UV (FUV) 


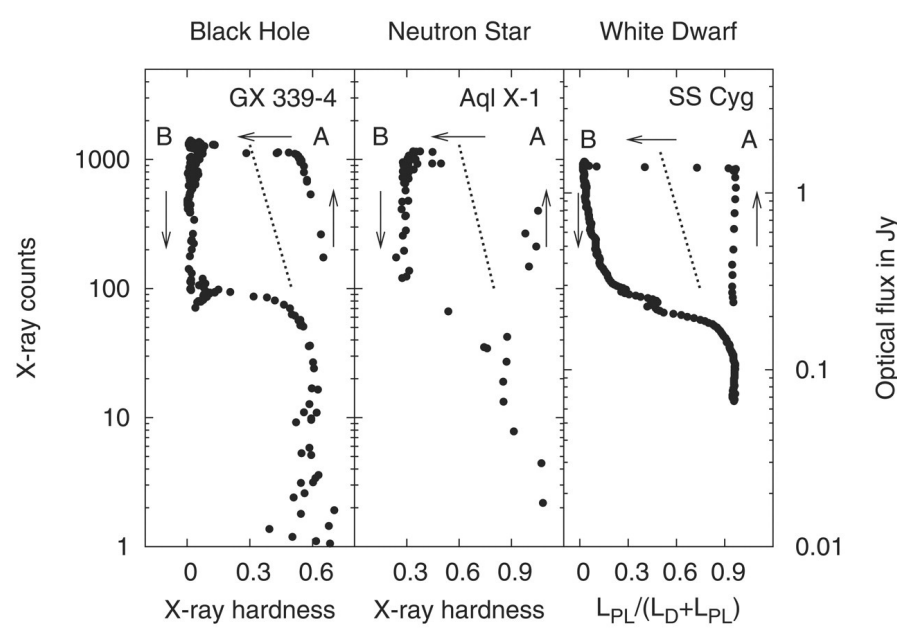

Fig. 1. Hardness-intensity diagrams for a black hole, neutron star, and cataclysmic variables. Here, $L_{\mathrm{PL}}$ and $L_{\mathrm{D}}$ are the X-ray and EUV luminosities, respectively. Data are taken from Maitra \& Bailyn (2004) and Wheatley et al. (2003). Figure reprinted from Körding et al. (2008) by permission of Science.

light curves and is usually described in terms of the so-called UV delay; the UV rise is delayed by approximately 0.5 day in a system such as SS Cyg (Cannizzo et al. 1986). It was initially thought that the importance of the delay was a good indicator of the place where the instability initially develops (Smak 1998): the so-called inside-out outbursts for which the instability develops close to the inner disc edge should exhibit shorter UV delays than the outside-in outbursts for which the instability sets in further away. Schreiber et al. (2003) showed that the situation is in fact more complex and found that disc truncation plays a major role: contrary to expectations, the delay for outside-in outbursts is usually shorter than for inside-out outbursts.

Körding et al. (2008) showed the temporal evolution of the optical flux versus the ratio of EUV to X-rays in SS Cyg: in quiescence, most of the flux is emitted in the X-ray band; during an outburst, the optical flux rises first; close to the outburst maximum, the X-ray flux vanishes while the EUV flux reaches its maximum; and during decline the optical flux decreases whereas the relative contribution of $\mathrm{X}$-rays increases up to its quiescent value. While this was not discussed in the paper, the data show a hysteresis when plotting the optical flux versus the EUV/X-ray hardness, which is very similar to that observed in low-mass $\mathrm{X}$-ray binaries (LMXBs); Fig. 1 in this work reproduces their Fig. 1. Körding et al. (2008) argued that the ratio of EUV to $\mathrm{X}$-rays is a measure of the optical depth of the inner parts of the accretion flow. They suggested that, similar to X-ray binaries (XRBs), CVs should emit the most prominent radio emission at the initial rise and during the transition from optically thin to optically thick inner accretion flow. In CVs, it is presumably the boundary layer that changes optical depth; in LMXBs, the transition from optically thin to optically thick most likely (certainly for LMXBs containing black holes) takes place in the disc. This is the phase during which the authors detected the source in radio bands.

The interpretation of the similarity between the HIDs for LMXBs and CVs is, however, not as straightforward as it might seem. LMXBs are also subject to the same instability as dwarf novae (King \& Ritter 1998; Dubus et al. 2001), which produce soft X-ray transients (SXTs; see e.g. Tanaka \& Shibazaki 1996; Chen et al. 1997; Yan \& Yu 2015; Tetarenko et al. 2016, for review of the observations). The differences between SXTs and
DNs are due to the nature of the compact object. The deep potential well results in strong irradiation of the accretion disc during SXT outbursts, and larger accretion discs are due to both a larger primary mass and longer orbital periods in LMXBs. These differences account for the much longer recurrence time between outbursts (years or decades instead of months) and longer outburst durations (months instead of a few days). The compactness of the accreting object is also responsible for most of the emission in SXTs to peak in the X-ray domain instead of the UV or EUV. In LMXBs, hard and soft X-rays originate from the innermost part of the accretion flow, whereas X-rays are emitted at the very vicinity of the white dwarf in cataclysmic variables, but optical light is emitted by the whole disc. The observed hysteresis therefore relates emission from the same region (or from very nearby regions) in LMXBs, whereas this is not at all the case in CVs.

In this paper, we reassess the multiwavelength time evolution of dwarf novae as predicted by the disc instability model with a particular emphasis on SS Cyg, for which we have the most comprehensive set of observations and for which the parameters (distance, orbital parameters) are reasonably well determined; we compare our results with observations. We also provide graphs showing where, according to the DIM, the majority of the emitted light originates as a function both of time and wavelength. We show that the DIM naturally accounts for the observed hysteresis when correlating X-ray and optical emission, whereas it cannot explain the hysteresis observed in LMXBs when plotting the X-ray flux versus the X-ray hardness. We show, however, that the existing data for SS Cyg suggest that a hysteresis could also exist when considering the X-ray plus EUV flow, which is presumably representative of the accretion rate in the inner disc versus the X-ray/EUV hardness ratio. This hysteresis, if confirmed, cannot be explained by the DIM alone; it would be similar to that observed in LMXBs and could point to similar mechanisms.

\section{The model}

We use the version of the DIM as described in Hameury et al. (1998) in which heating by the tidal torque and stream impact have been incorporated (Buat-Ménard et al. 2001). We also take into account irradiation of the accretion disc by the compact object, which impacts the outburst cycle by modifying the vertical disc structure and thus the S-curve and the emitted spectra owing to reprocessing. For the spectral modelling, we follow the procedure described in Schreiber et al. (2003) with the difference that the disc spectrum is obtained by summing black bodies instead of Kurucz (1979) spectra. Given the small differences found in Schreiber et al. (2003) between these two different approximations, and given the fact that the spectrum of an accretion disc is not very well represented locally by a Kurucz spectrum, this is a simple and fair assumption. A detailed calculation, as carried out in Idan et al. (2010) for time-dependent accretion discs, would be CPU intensive and clearly outside of the scope of this paper.

In addition to the flux emitted by the accretion disc, we take into account the contribution of the boundary layer, which is assumed to emit hard X-rays when optically thin; we assume that this happens when the accretion rate is below $2 \times 10^{16} \mathrm{~g} \mathrm{~s}^{-1}$. This value is twice as high as that used in Schreiber et al. (2003) to account for the smaller white dwarf mass and hence that larger emitting surface that we assume here (see below). In order to obtain a smooth transition, we assume that the X-ray flux is cut by an exponential factor $\exp \left(-\dot{M}_{\text {acc }} / 2 \times 10^{16} \mathrm{~g} \mathrm{~s}^{-1}\right)$. When optically thick, the spectrum is that of a black body, and we 
Table 1. Binary parameters for SS Cyg.

\begin{tabular}{lcc}
\hline \hline Parameter & Value & References \\
\hline$P_{\text {orb }}(\mathrm{h})$ & 6.603 & 1 \\
$M_{\mathrm{wd}}\left(M_{\odot}\right)$ & 1.00 & 2 (see text) \\
$M_{2} / M_{\mathrm{wd}}$ & 0.67 & 2 \\
$R_{\text {tid }}(\mathrm{cm})$ & $5.17 \times 10^{10} \mathrm{~cm}$ & \\
$R_{\mathrm{wd}}(\mathrm{cm})$ & $5.5 \times 10^{8} \mathrm{~cm}$ & \\
Distance $(\mathrm{pc})$ & 114 & 3 \\
\hline
\end{tabular}

References. (1) Ritter \& Kolb (2003); (2) Bitner et al. (2007); (3) Miller-Jones et al. (2013).

assume that the emitting area is $f_{\mathrm{em}} 4 \pi R_{\mathrm{wd}}^{2}$, with $f_{\mathrm{em}}$ given by (Patterson \& Raymond 1985; Schreiber et al. 2003)

$f_{\mathrm{em}}=10^{-3}\left(\frac{\dot{M}_{\mathrm{acc}}}{10^{16} \mathrm{~g} \mathrm{~s}^{-1}}\right)^{0.28}$,

where $R_{\text {wd }}$ is the white dwarf radius and $\dot{M}_{\text {acc }}$ is the accretion rate onto the white dwarf.

We also include the contribution of the white dwarf, which is assumed to be a black body with a constant temperature, and the contribution of the hot spot luminosity, which is not included in the disc model; we assume that the hot spot radiates as a black body with a temperature of $10000 \mathrm{~K}$. We assume that one-half of the stream impact energy is emitted by the hot spot. We finally include the contribution from the secondary star, which is the sum of two black bodies with different effective temperatures but the same emitting area: the unilluminated backside of the secondary, plus the contribution of the illuminated side of the secondary, which is assumed to have a temperature $T_{2}$ given by

$T_{2}^{4}=T_{*}^{4}+\left(\frac{R_{\mathrm{wd}}}{a}\right)^{2}\left(T_{\mathrm{BL}}^{4} f_{\mathrm{em}}+T_{\mathrm{wd}}^{4}\right)$,

where $T_{*}, T_{\mathrm{BL}}$, and $T_{\mathrm{wd}}$ are the secondary, boundary layer, and white dwarf temperatures, respectively, and $a$ is the orbital separation. Irradiation by the disc is not included in Eq. (2) since the disc luminosity is emitted perpendicular to the disc plane and only a small fraction of it can contribute to heating of the secondary. The unilluminated temperature is taken to be $4000 \mathrm{~K}$. As for the disc, one could have assumed the secondary spectrum to be given by Kurucz (1979). Again, this refinement is not appropriate given the approximations made here and, in particular, given the fact that the temperature of the irradiated side of the secondary is constant over the whole hemisphere instead of decreasing from the equator to the pole; we therefore use a black-body spectrum.

We use the orbital parameters given in Table 1. We have chosen $M_{1}=1 M_{\odot}$, which does not correspond to the fiducial value given by Bitner et al. (2007) of $0.81 M_{\odot}$ and is significantly smaller than the previously used value of $1.2 M_{\odot}$ because with a primary mass of $0.81 M_{\odot}$, the disc extension is small and the peak luminosity cannot reach the observed value. The mass $M_{1}=1.0 M_{\odot}$ corresponds to the upper range of allowed values [0.62-1.00] $M_{\odot}$ provided by Bitner et al. (2007). The value of the radio parallax determined by Miller-Jones et al. (2013) also requires that the primary mass to be close to the mass of the Sun.

\section{Results for accreting white dwarfs}

We first consider the case where the disc is truncated by, for example a magnetic field, and does not extend all the way down

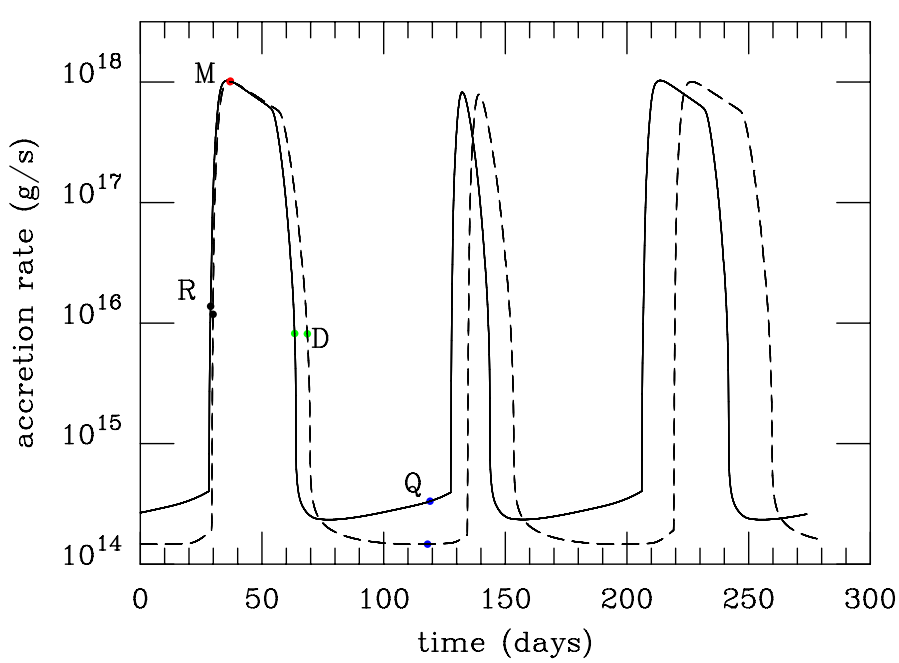

Fig. 2. Light curve obtained using parameters given in Table 1. The solid line corresponds to the unilluminated case; the dashed line to the maximally irradiated case. Snapshots in Figs. 3-5 are taken at phases labelled R, M, D, and Q.

to the white dwarf surface during quiescence, which provides the best fit for the UV delay in SS Cyg (Schreiber et al. 2003). We take the truncation radius to be

$r_{\text {in }}=5.21 \times 10^{8} \mu_{30}^{4 / 7} M_{1}^{-1 / 7}\left(\frac{\dot{M}_{\mathrm{acc}}}{10^{16} \mathrm{~g} \mathrm{~s}^{-1}}\right)^{-2 / 7} \mathrm{~cm}$,

which corresponds to the case where truncation is caused by a dipolar magnetic field with moment $\mu_{30}$ in units of $10^{30} \mathrm{G} \mathrm{cm}^{3}$, and $M_{1}$ is the primary mass in solar units.

We consider two extreme cases for the irradiation of the disc: the case where irradiation is neglected and the case where it is treated as in Hameury et al. (2000), i.e. the irradiation flux impinging onto the disc is given by:

$\sigma T_{\mathrm{ill}}^{4}=\frac{G M_{\mathrm{wd}} M_{\mathrm{acc}}^{3}}{8 \pi R_{\mathrm{wd}}} \frac{1}{\pi}\left[\arcsin \rho-\rho\left(1-\rho^{2}\right)^{1 / 2}\right]$,

where $\rho=R_{\mathrm{wd}} / r$ and $r$ is the radial coordinate in the disc. This assumes that the energy released by accretion onto the white dwarf is emitted isotropically by the white dwarf surface. It is a fair approximation when the disc is truncated. It clearly is an overestimate when an optically thick boundary layer forms, since only a fraction of this energy is thermalized by the whole white dwarf surface. Irradiation at large distances by a hot white dwarf decreases as $r^{-3}$; this irradiation is very significant in the inner parts of the disc because the white dwarf extends very much above the disc surface, but the outer parts of the disc are not much affected by irradiation. This contrasts with the LMXB case, for which this effect does not exist. In LMXBs, irradiation is observed to be responsible for most of the optical light during outbursts or in steady systems. In this case irradiation can vary as $r^{-2}$, but with a relatively small coefficient (Dubus et al. 1999 assume that $\sigma T_{\text {ill }}^{4}=C L_{\mathrm{x}} / 4 \pi r^{2}$ with $\left.C \sim 5 \times 10^{-3}\right)$. As viscous heating varies as $r^{-3}$, irradiation always overcomes viscous heating at large distances if the disc is large enough. This is the case in LMXBs; in CVs, using the same value for $C$ does not lead to significant effects because of the smaller disc extent and the smaller accretion efficiency. Our numerical simulations have shown that this is indeed the case.

The resulting light curve is presented in Fig. 2. It shows an alternation of long and short outbursts, as observed in SS Cyg. 


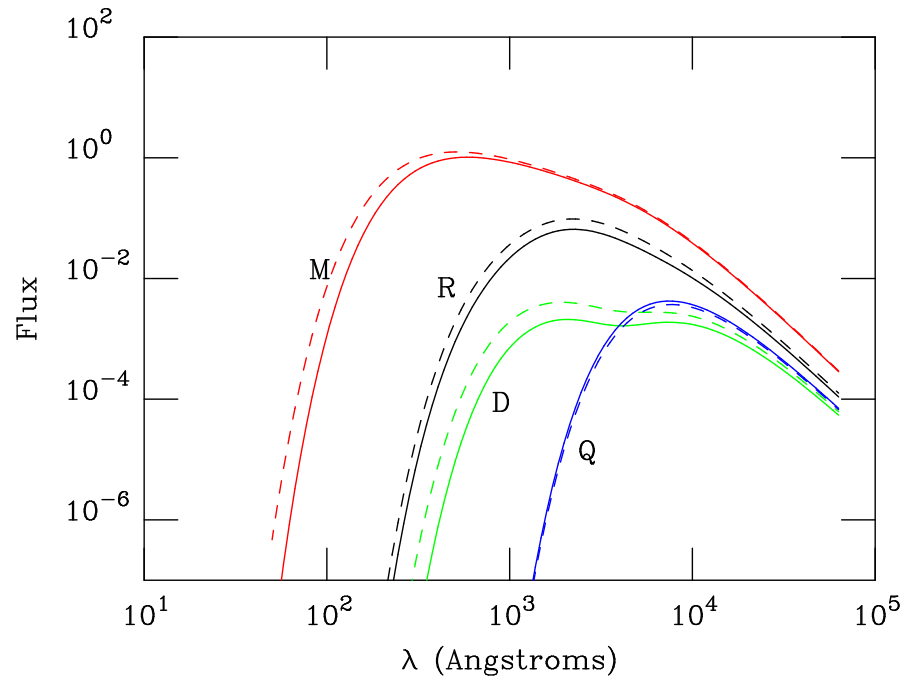

Fig. 3. Energy spectra emitted by the accretion disc at times indicated in Fig. 2. The black curve corresponds to the rise to maximum (R), the red curve to the outburst maximum (M), the green curve to decay (D), and the blue curve to quiescence (Q). The solid line corresponds to the unilluminated case; the dashed line to the maximally irradiated case. Contributions from other components, i.e. white dwarf surface, boundary layer, hot spot, and secondary star, have not been included here.

The irradiated and unirradiated cases are very similar; when irradiation is taken into account, the major outbursts last slightly longer, the quiescence level is reduced because the disc mass is lower at the end of an outburst, and the recurrence time is slightly increased. We show the disc spectra obtained at four different epochs in Fig. 3, which is representative of the rise to maximum, maximum, decay, and quiescence. Contributions from other components of the system (white dwarf, secondary, boundary layer, and hot spot) have not been included here; except for the boundary layer, their contribution is significant only during quiescence. Irradiation has little effect on these spectra; the main differences are that they extend to slightly lower wavelengths and the fluxes are slightly higher in the irradiated case even though the accretion rate is almost identical, except in quiescence. Figures 4 and 5 show the contribution of each ring in the disc to the UV (1250 ̊) and optical (5500 $⿱$ A) fluxes. The position of the maximum in $r^{2} F_{v}$ indicates the region of the disc, which is the main contributor to the flux, provided that $F_{v}(r)$ varies on scales of order of $r$, which is obviously not the case for the outermost regions of the disc, where heating is dominated by dissipation of tidal forces. Despite the high value of $r^{2} F_{v}$ at the outer edge, these regions contribute less than $1 \%$ to the total UV flux throughout the whole cycle and to $10 \%$ of the optical flux at maximum but $60 \%$ during decay. As can be seen, in the hot state, the UV-domain $r^{2} F_{v}$ curve is rather flat over most of the disc and all regions contribute more or less evenly to the UV flux. This contrasts with the optical wavelengths where most of the flux originates from the outermost ring in the hot state. It can also be seen that differences between the irradiated and nonirradiated cases exist in optical during decay. They appear in regions of the disc that have returned to the cold state and do not contribute much to the optical light anyway. Differences are also in the contributions of the inner disc to the optical light during quiescence, which are also small; these are consistent with the reduced $\dot{M}_{\text {acc }}$ in the irradiated case.

These results could in principle be compared with observations of dwarf novae using eclipse mapping techniques (see e.g.

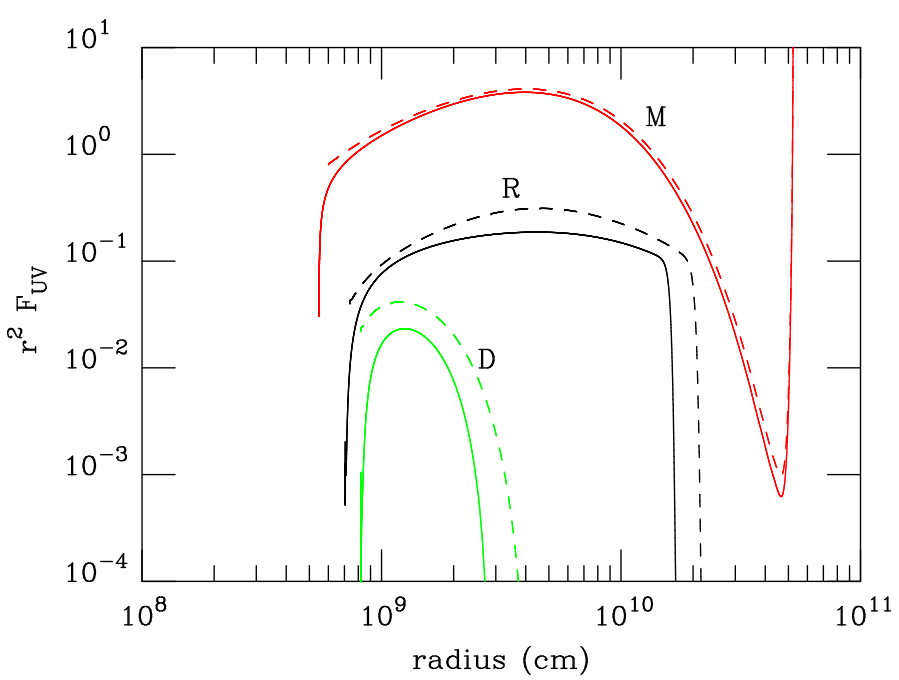

Fig. 4. Radial distribution of the UV flux during rise, maximum, and decay. The colour coding is the same as in Fig. 5. The solid line corresponds to the unilluminated case; the dashed line to the maximally irradiated case. The UV flux in quiescence is vanishingly small and is not shown here.

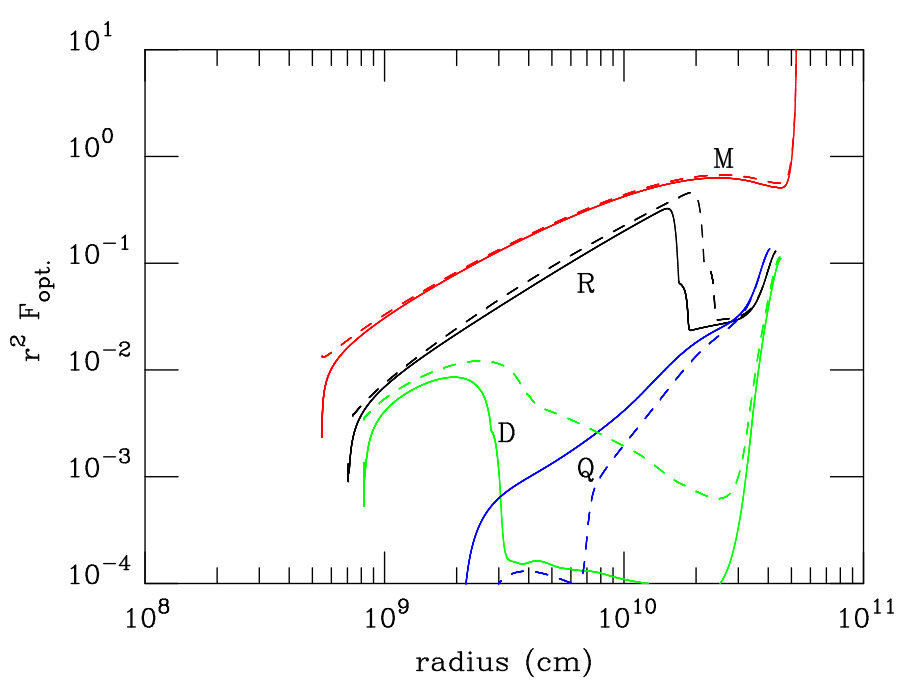

Fig. 5. Radial distribution of the optical flux at times shown in Fig. 2. The black curve corresponds to the rise to maximum (R), the red curve to the outburst maximum (M), the green curve to decay (D), and the blue curve to quiescence $(\mathrm{Q})$. The solid line corresponds to the unilluminated case; the dashed line corresponds to the maximally irradiated case.

Baptista 2016, and references therein). The examination of Fig. 3 in Baptista \& Catalán (2001) representing the radial distribution of the intensity of the dwarf nova EX Dra shows, however, a number of differences with the picture presented here. In particular, in quiescence, most of the optical light is emitted by the innermost part of the accretion disc with a significant, though smaller, contribution from an outer ring that is not axisymmetric. One should keep in mind that, because this technique is limited to high inclination systems, some artefacts may be present (Smak 1998). Irradiation of the inner disc by the white dwarf can also be significant during quiescence and has not been included here.

Figure 5 shows that the disc luminosity at time R during the rise phase is much higher than the luminosity at time $\mathrm{D}$ during decay; yet, the accretion rates at the inner disc edge are comparable in both cases. The reason for this is that during the rise, the accretion rate at the inner edge of the disc increases on a 


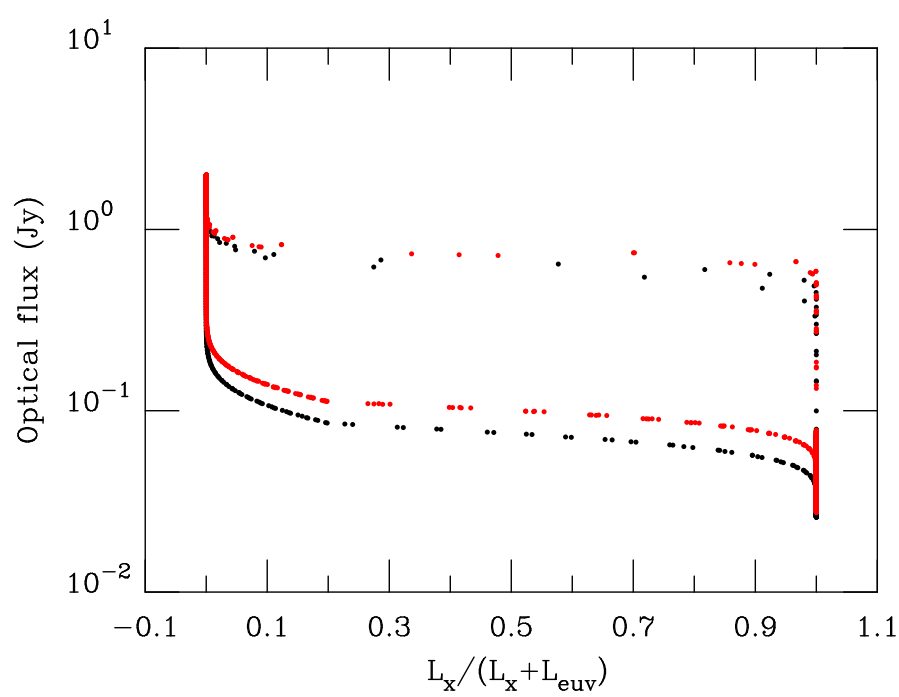

Fig. 6. Hardness intensity diagram from the DIM. Several outbursts cycles are represented here, and each point is separated by approximately $3 \mathrm{~h}$. The unirradiated case is shown in black; the irradiated case is shown in red.

viscous time, while the heating front propagates outwards on a much shorter thermal time. On the other hand, during the decay phase, the cooling front propagates inwards at a slower speed than a heating front (Menou et al. 1999). For a given disc optical luminosity, and hence a given position of the transition front, the accretion rate should be lower during rise than during the decay. It is therefore not surprising that a hysteresis, such as that seen by Körding et al. (2008), is found in dwarf nova outbursts.

Figure 6 shows the hardness versus intensity diagram expected for a dwarf nova such as SS Cyg as predicted by the DIM. Here, the $x$-axis is the ratio between the X-ray and the X-ray-plus-EUV (defined as $\lambda$ smaller than $130 \AA$ ) luminosities. Several outbursts are represented on this diagram, and both the long and short outbursts follow the same track even though the accreted mass is different in each case; $10 \%$ versus $40 \%$ of the total disc mass is accreted during short and long outbursts, respectively. Our simulations have shown that short outbursts would produce different tracks on such a diagram only if the heating front were not able to reach the outer edge of the disc and the peak luminosity was much smaller than for long outbursts, which is not the case for SS Cyg. We found that this happens when, for example the primary mass is large and the truncation radius is small. The irradiated and unirradiated cases are very similar; irradiation slightly reduces the amplitude of the hysteresis because some optical flux, which is proportional to $\dot{M}_{\text {acc }}$ (and hence depending only on the ratio $L_{\mathrm{X}} /\left(L_{\mathrm{X}}+L_{\mathrm{euv}}\right)$ ), is added. But because irradiation is negligible in the outer parts of the disc that contribute to most of the optical flux, this effect is small.

This figure can be directly compared with Fig. 1 from Körding et al. (2008). The general characteristics of our modelgenerated diagrams are rather similar to the observational diagram. The maximum optical luminosity is, as expected, close to the observed value. The transition between X-ray and EUV emission on the upper branch occurs at about the right optical luminosity, which means that the critical $\dot{M}_{\text {acc }}$ for the transition between optically thin and optically thick X-ray emission is not very different from the value used here $\left(2 \times 10^{16} \mathrm{~g} \mathrm{~s}^{-1}\right)$. A slightly higher value would have given an even better agreement. We also, note that the transition is very sharp. The optical

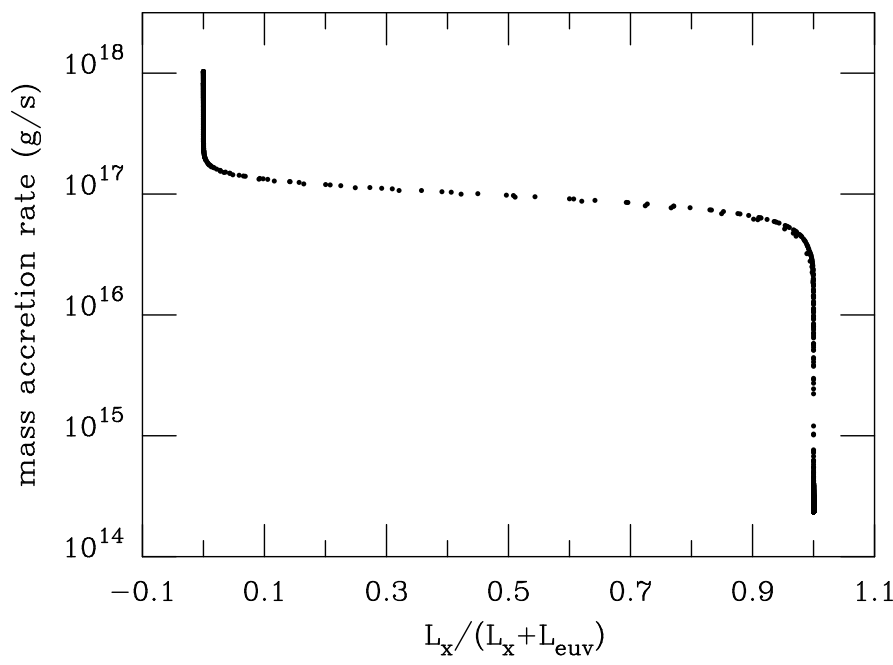

Fig. 7. Hardness mass-accretion-rate onto the compact object diagram. As in Fig. 6, several cycles are plotted on this diagram. Such a figure also represents the predictions of the DIM alone for low-mass X-ray binaries.

luminosity on the lower branch is also quite comparable to the observed luminosity, however, the transition on the lower branch is smoother than that on the upper branch, but not quite as smooth as observed. This means that the exponential cut-off of the X-ray luminosity is probably too sharp.

In order for this comparison to be meaningful, the spectral model has to be as realistic as possible. Modelling the contribution of the inner and outer disc and of the boundary layer is relatively simple in contrast with the LMXB case (see below). It should be stressed that the hysteresis would appear in any case when considering the optical luminosity and any quantity linked to the mass accretion rate at the inner edge of the disc, and the conclusion that the DIM is responsible for the observed hysteresis is in fact very solid and does not depend on the detailed spectral modelling of the inner flow.

Figure 7 shows the same diagram as Fig. 6, but in Fig. 7 we plot the mass accretion rate onto the white dwarf instead of the optical luminosity. As can be seen, the hysteresis has disappeared and there is a univocal relation between $\dot{M}_{\text {acc }}$ and the hardness ratio. This is merely because the innermost part of the system that emits X-rays and EUV has very short characteristic timescales and are therefore in a quasi-steady-state situation with a local mass transfer rate determined by the outer parts of the disc that have a much longer viscous time. The precise shape of the dependence between $\dot{M}_{\text {acc }}$ and the hardness ratio depends on the various assumptions made for the boundary layer and is therefore quite uncertain; but the very existence of the hysteresis in the hardness - optical luminosity diagram is not affected by these assumptions.

For the sake of completeness, we also calculated the hardness intensity diagram, which we obtain when we do not assume that the disc is truncated in quiescence, all other parameters being identical. The light curves we find are relatively different from the truncated case: we obtain only one type of outburst, which is similar to the short outburst shown in Fig. 2 and separated by 47 days; the peak optical luminosity is lower. Yet the hardness intensity diagrams we get in both cases are almost identical. This should not come as a surprise, since this diagram is largely determined by the viscous time in the outer parts of the disc, which is responsible for the delay between the rise in the optical and the increase of the accretion rate onto the white dwarf. 


\section{The case of LMXBs}

The DIM provides a natural explanation for the occurrence of SXT outbursts in LMXBs. But the DIM focus is essentially on the outer regions of the disc, where the effective temperature is of the order of a few thousands degrees and where the instability occurs. Moreover, standard geometrically thin, optically thick Shakura-Sunyaev accretion discs are unable to produce the observed hard X-rays observed in LMXBs. The formation of an advection dominated flow when the mass transfer rate is low (ADAF, Esin et al. 1997) is an option which got strong support when realizing that the accretion disc had to be truncated for the DIM to be applicable to SXTs (Dubus et al. 2001). It remains, however, that there is no widely accepted model that can account for the spectral transitions of LMXBs, and henceforth for providing theoretical spectra of accretion discs for given physical parameters (see e.g. Salvesen et al. 2013; Nixon \& Salvesen 2014, for recent alternative models).

In addition, modelling the HID for LMXBs requires, in contrast with the CV case, an accurate modelling of the spectra of each of the components of the flow. This is because the energy bands used to determine the hardness ratio are adjacent, whereas in the CV case the EUV and X-ray bands are well separated and originate from well-defined regions. And indeed, spectral models of accretion discs incorporating the effects of local dissipation in the vertical structure of the disc differ significantly from black bodies (Tao \& Blaes 2013).

Despite these difficulties, it is, however, simple to show that the hysteresis in LMXBs is different from that observed in CVs. The viscous time in the regions of the accretion disc where X-ray are produced is

$t_{\mathrm{visc}}=\frac{r^{2}}{v}=\frac{1}{\alpha} \frac{v_{\mathrm{K}}^{2}}{c_{\mathrm{s}}^{2}}=\frac{1}{2 \alpha} \frac{c^{2}}{c_{\mathrm{s}}^{2}} \frac{r_{\mathrm{s}}}{r}$,

where $v$ is the viscosity, $v_{\mathrm{K}}$ is the Kepler viscosity, and $r_{\mathrm{S}}$ is the Schwartzschild radius. For X-rays to be emitted, the effective temperature has to be higher than $10^{7} \mathrm{~K}$, and hence the viscous time is less than

$t_{\mathrm{visc}}<10^{5} \frac{1}{\alpha} \frac{r_{\mathrm{s}}}{r} \mathrm{~s}$,

i.e. is always less than a day or a few days. On the transient timescale, the inner part of the disc is therefore in a quasi-steadystate situation, where a local mass transfer is imposed by the external parts of the disc that evolve on a much longer viscous time. This then requires that additional mechanisms are responsible for the existence of two different accretion flows for a given X-ray luminosity. Many such mechanisms have been proposed (see e.g. Begelman \& Armitage 2014; Nixon \& Salvesen 2014; Kylafis \& Belloni 2015; Cao 2016, for recent examples); they are clearly disconnected from the DIM.

\section{SS Cyg: a hysteresis in the $L_{\mathrm{X}}-L_{\mathrm{EUV}}$ domain?}

The DIM-based explanation of the hysteresis observed by Körding et al. (2008) does not mean that a hysteresis similar to that observed in LMBXs does not exist in CVs, especially considering that for SS Cyg the white-dwarf jet-formation criterion by Soker \& Lasota (2004) is not satisfied. But such a hysteresis should relate quantities characterizing the state of the inner disc. This is, however, difficult to observe because one needs to measure the accretion rate at the inner edge, while most of the related emission is in the UV - EUV band; thus this emission

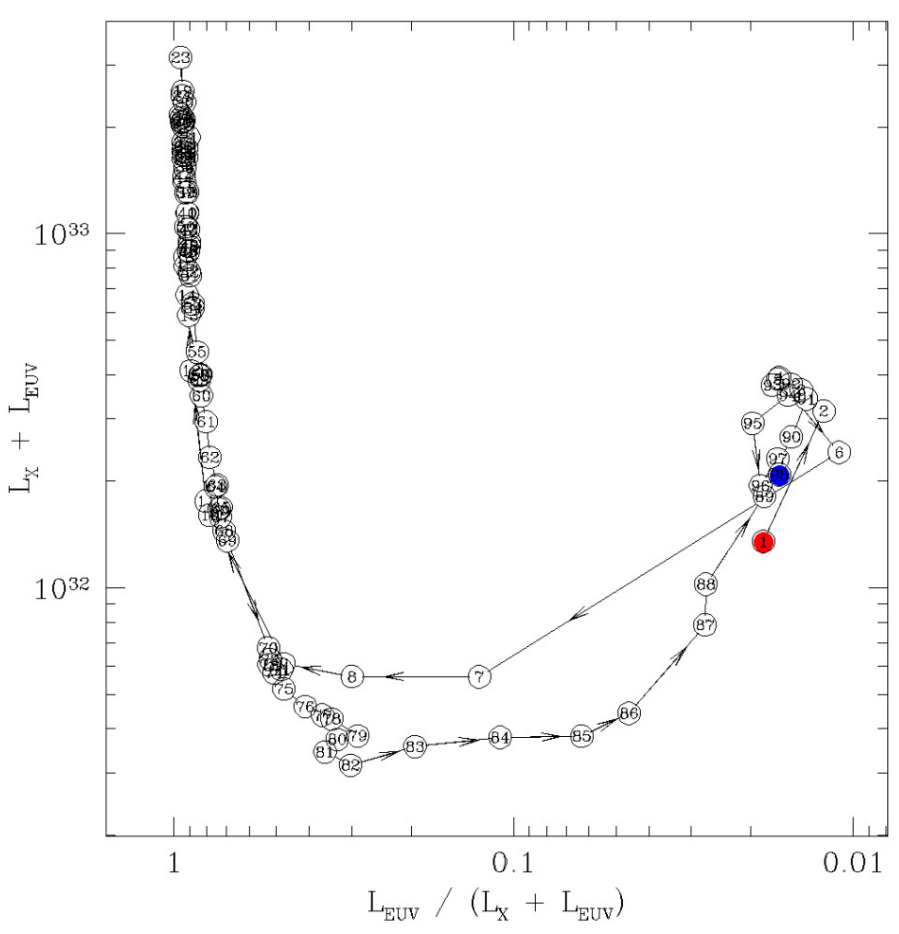

Fig. 8. Observed HID for SS Cyg when considering the EUV - X-ray domain.

is heavily absorbed and the bolometric corrections are important and uncertain.

In Fig. 8 we show the HID obtained for SS Cyg when plotting the EUV luminosity versus the hardness defined as the ratio of the EUV and X-ray plus EUV luminosities, which should be representative of the accretion rate onto the white dwarf. The data are taken from Wheatley et al. (2003). Unfortunately the hysteresis hinges on two data points, but has a magnitude that is larger than 0.3 dex. A similar picture can be seen if one plots the X-rays as a function of hardness. If one combines both the $\mathrm{X}$-rays and EUV to get something like a bolometric luminosity, one obtains a HID that is not dissimilar to HIDs seen in XRBs. As we do not know the variable bolometric correction factors, it is unclear how these plots relate to a proper HID for the inner accretion flow. But the data are indicative that such a hysteresis can exist. If it were confirmed, such a hysteresis would not be related to the DIM, as shown by Fig. 7, and would in fact bring no constrain on the instability responsible for dwarf nova outbursts; any mechanism accounting for the outburst of dwarf nova outburst (such as e.g. the mass transfer instability model; Bath 1973) would produce exactly the same HID because the inner disc is quasi-steady. This hysteresis would, however, be key in understanding the physics of accretion in the immediate vicinity of the white dwarf.

\section{Conclusions}

The DIM naturally produces a hysteresis in a diagram plotting the optical luminosity versus the X-ray to EUV hardness or any quantity that is directly related to the accretion rate onto the white dwarf. This hysteresis simply occurs because it takes a viscous time for the mass transfer rate to vary at the inner disc edge whereas the optical luminosity varies on the much shorter thermal time. This is very different from the LMXB case in which the hysteresis relates soft and hard X-rays that are both emitted in the innermost parts of the disc; on observational timescales, 
these inner parts of the disc can safely be assumed to be in a steady-state situation, meaning that for a given local mass accretion rate, two quasi-stable solutions must exist. In addition, in $\mathrm{CVs}$ the hysteresis relates emission from various parts of the accretion disc whereas in case of LMXBs it might relate emission from the disc and from a hot extended flow that is not part of the disc.

The X-ray - EUV hardness-intensity diagram for SS Cyg, which is similar to the classical HID for LMXBs, gives a clear indication that a hysteresis is present. The DIM cannot account for this hysteresis, which is indicative of the presence of several components within the accretion flow for a given mass accretion rate in the inner disc, and whose existence depends on the history of the system. This hysteresis, however, needs to be confirmed.

It is difficult, but not impossible, to test the models with current instrumentation. The major difficulty is accessing the EUV (CVs) and/or soft X-rays (XRBs). There are two problems there: first, they are so easily absorbed away by the interstellar medium (ISM); second, there are few observatories and instruments capable of observing in this regime. Overcoming the ISM issue, which especially affects the EUV, requires observing systems specifically selected to have low NH columns. Such systems are rare, but they do exist. Perhaps the best example of such a system is VW Hyi, which has a column of only $N_{\mathrm{H}}=6 \times 10^{17} \mathrm{~cm}^{-2}$ (Polidan et al. 1990). There are also others that may in principle be observable (e.g. SS Cyg was successfully observed with EUVE). Overcoming the instrumental issue is harder; the EUVE no longer exists. Of the currently available space observatories and instruments, the only one that could be used for CVs in this regard is the Chandra/LETG combination. In fact, this has already been used to study WZ Sge (Mauche 2004a) and SS Cyg (Mauche 2004b). The Great Observatories Accretion Legacy Survey (GOALS) is an observational campaign being developed, which is aimed at following an entire dwarf nova outburst from rise to decline across all wavelengths, covering X-rays, EUV, FUV, NUV, optical, NIR, and radio. A GOALS-Pathfinder campaign with Chandra, which is aimed at establishing whether two candidate targets (RX And and $\mathrm{YZ} \mathrm{Cnc)}$ ) are detectable with the LETG, is already approved and underway.

Acknowledgements. This work was supported by a National Science Centre, Poland grant 2015/19/B/ST9/01099. J.P.L. was supported by a grant from the French Space Agency CNES.

\section{References}

Bailey, J. 1980, MNRAS, 190, 119

Balbus, S. A., \& Hawley, J. F. 1991, ApJ, 376, 214

Bath, G. T. 1973, Nat. Phys. Sci., 246, 84

Bath, G. T., \& Pringle, J. E. 1981, MNRAS, 194, 967

Baptista, R. 2015, in Astronomy at High Angular Resolution, A compendium of techniques in the visible and near-infrared, Springer ASSL series 439, eds. H. M. J. Boffin, G. Hussain, J. P. Berger., \& L. Schmidtobreick [arXiv: 1508.03067]

Baptista, R., \& Catalán, M. S. 2001, MNRAS 324, 599

Begelman, M. C., \& Armitage, P. J. 2014, ApJ, 782, L18

Bitner, M. A., Robinson, E. L., \& Behr, B. B. 2007, ApJ, 662, 564

Buat-Ménard, V., Hameury, J. M., Lasota, J. P. 2001, A\&A, 366, 612

Cannizzo, J. K., Wheeler, J. C., \& Polidan, R. S. 1986, ApJ, 301, 634

Cao, X. 2016, ApJ, 817, 71

Chen, W., Shrader, C. R., \& Livio, M. 1997, ApJ, 491, 312

Coleman, M. S. B., Kotko, I., Blaes, O., Lasota, J.-P., \& Hirose, S. 2016, MNRAS, 462, 3710

Dubus, G., Lasota, J.-P., Hameury, J.-M., \& Charles P. 1999, MNRAS, 303, 139

Dubus, G., Hameury, J.-M., \& Lasota, J.-P. 2001, A\&A, 373, 251

Esin, A. A., McClintock, J. E., \& Narayan, R. 1997, ApJ, 489, 865

Hameury, J.-M., Menou, K., Dubus, G., Lasota, J.-P., \& Hure, J.-M. 1998, MNRAS, 298, 1048

Hameury, J.-M., Lasota, J.-P., \& Warner, B. 2000, A\&A, 353, 244

Idan, I., Lasota, J.-P., Hameury, J.-M., \& Shaviv, G. 2010, A\&A, 519, A117

King, A. R., \& Ritter, H. 1998, MNRAS, 293, L42

Körding, E., Rupen, M., Knigge, C., et al. 2008, Science, 320, 1318

Kylafis, N. D., \& Belloni, T. M. 2015, A\&A, 574, A133

Kurucz, R. 1979, ApJS, 40, 1

Lasota, J.-P. 2001, New Astron. Rev., 45, 449

Maitra, D., \& Bailyn, C. D. 2004, ApJ, 608, 444

Mauche, C. W., 2004b, Rev. Mex. Astron. Astrofís., 20, 174

Mauche, C. W., 2004b, ApJ, 610, 422

Menou, K., Hameury, J. M., \& Stehle, R. 1999, MNRAS, 305, 79

Miller-Jones, J. C. A., Sivakoff, G. R., Knigge, C., et al. 2013, Science, 340, 950

Nixon, C., \& Salvesen, G. 2014, MNRAS, 437, 3994

Patterson, J., \& Raymond, J. C. 1985, ApJ, 292, 550

Polidan, R. S., Mauche, C. W., \& Wade, R. A. 1990, ApJ, 356, 211

Ritter, H., \& Kolb, U. 2003, A\&A, 404, 301

Salvesen, G., Miller, J. M., Reis, R. C., \& Begelman, M. C. 2013, MNRAS, 431, 3510

Schreiber, M. R., Hameury, J. M., Lasota, J. P. 2003, A\&A, 410, 239

Shakura, N. I., \& Sunyaev, R. A. 1973, A\&A, 24, 337

Smak, J. 1994, Acta Astron., 44, 265

Smak, J. 1998, Acta Astron., 48, 677

Soker, N., \& Lasota, J.-P. 2004, A\&A, 422, 1039

Tanaka, Y., \& Shibazaki, N. 1996, ARA\&A, 34, 607

Tetarenko, B. E., Sivakoff, G. R., Heinke, C. O., \& Gladstone, J. C. 2016, ApJS, 222,15

Tao, T., \& Blaes, O. 2013, ApJ, 770, 55

Warner, B. 1995, Cataclysmic variable stars, Camb. Astrophys. Ser., 28

Wheatley, P. J., Mauche, C. W., \& Mattei, J. A. 2003, MNRAS, 345, 49

Yan, Z., \& Yu, W. 2015, ApJ, 805, 87 